PROCEEDINGS OF THE

AMERICAN MATHEMATICAL SOCIETY

Volume 130, Number 1, Pages 145-156

S 0002-9939(01)06148-2

Article electronically published on June 8, 2001

\title{
SINGULAR CONTINUOUS SPECTRUM FOR A CLASS OF NONPRIMITIVE SUBSTITUTION SCHRÖDINGER OPERATORS
}

\author{
CÉSAR R. DE OLIVEIRA AND MARCUS V. LIMA
}

(Communicated by Joseph A. Ball)

\begin{abstract}
We present a class of discrete Schrödinger operators, with potentials derived from nonprimitive substitutions, that has purely singular continuous spectrum. We give sufficient conditions on the substitution rule assuring singular continuous spectrum, either for a generic set in the hull of the potential or for a set of total invariant measure.
\end{abstract}

\section{InTRODUCTION AND RESUlts}

From the topological point of view the "building blocks" of dynamical systems are the minimal ones, i.e., those for which all orbits are dense; from the measure theoretical point of view such building blocks are the uniquely ergodic systems, i.e., those with just one probability invariant measure. In some cases these two properties are simultaneously verified and such dynamical systems are called strictly ergodic, a property that may present deep consequences. An important recent application of this concept is on the spectral study of a class of (discrete) Schrödinger operators, whose potentials take finite many values, generated by primitive substitution rules, with the predominance of pure singular continuous spectrum [1]-16]. In this work we give new examples of Schrödinger operators with substitution potentials with purely singular continuous spectrum, but here we show that many techniques used to investigate the primitive case (including strict ergodicity) can be adapted to a class of nonprimitive substitutions; it expands and generalizes results for a particular substitution presented in our previous paper [17. We also mention that, from the physical point of view, the substitution Schrödinger operators are quantum one-dimensional models of quasicrystals, whose potentials are neither random nor periodic, but exhibit self-similarities.

Let's clarify what is called a substitution potential and primitivity. Since we consider only potentials taking two values we shall restrict our discussion to substitutions $\xi: \mathcal{A} \mapsto \mathcal{A}^{*}$ on an alphabet $\mathcal{A}=\{a, b\} ; \mathcal{A}^{*}$ is the set of all words of finite length on the alphabet. If we denote by $\mathcal{A}^{\mathbb{N}}$ the set of all sequences of letters in $\mathcal{A}$, the substitution $\xi$ have natural extensions, by concatenation, to $\mathcal{A}^{*}$ and $\mathcal{A}^{\mathbb{N}}$; for example, $\xi(a b)=\xi(a) \xi(b)$. A substitution sequence is a fixed point $\bar{\eta}$ of $\xi$ in $\mathcal{A}^{\mathbb{N}}$, i.e., $\xi(\bar{\eta})=\bar{\eta}$. We consider on $\mathcal{A}^{\mathbb{N}}\left(\mathcal{A}^{\mathbb{Z}}\right)$ the point convergence topology generated

Received by the editors May 30, 2000.

1991 Mathematics Subject Classification. Primary 81Q10; Secondary 11B85, 47B39.

The first author was partially supported by CNPq (Brazil). 
by the metric

$$
d(u, v)=\sum_{n} \frac{\left|u_{n}-v_{n}\right|}{2^{|n|}}, u=\left(u_{n}\right), v=\left(v_{n}\right),
$$

with $n \in \mathbb{N}(n \in \mathbb{Z})$. Then, we take $a$ as a seed and suppose that $\xi(a)$ begins with $a$ and that the length of $\xi^{k}(a)$ goes to infinity as $k \mapsto \infty\left(\xi^{k}\right.$ denotes the $k$-th iterate of $\xi$ ), assuring the existence of a fixed point for $\xi$ (see [19, Proposition V.1).

Given a substitution sequence $\bar{\eta}$, consider the periodic sequences in $\mathcal{A}^{\mathbb{Z}}$

$$
\eta_{n}=\cdots \xi^{n}(a) \xi^{n}(a) \cdot \xi^{n}(a) \xi^{n}(a) \cdots,
$$

with the dot indicating the position of the term of index zero. If $\xi(a)$ begins and ends with $a$, then $\left(\eta_{n}\right)$ is a Cauchy sequence and one gets a well-defined limit sequence

$$
\eta=\lim _{n \rightarrow \infty} \eta_{n}
$$

in $\mathcal{A}^{\mathbb{Z}}$, called the bilateral substitution sequence generated by $\xi$. Such conditions will be satisfied by the substitution class $\zeta_{N}$ that will be considered in this work.

Let $T: \mathcal{A}^{\mathbb{Z}} \mapsto \mathcal{A}^{\mathbb{Z}}$ be the left shift $(T u)_{n}=u_{n+1}$; the hull of $\eta$ in $\mathcal{A}^{\mathbb{Z}}$ is defined as

$$
\Omega=\Omega(\eta)=\operatorname{closure}\left\{T^{n} \eta: n \in \mathbb{N}\right\} .
$$

We notice that for a recurrent sequence $\eta$ its hull $\Omega$ is a compact and $T$-invariant subset of $\mathcal{A}^{\mathbb{Z}}$ with $T \Omega(\eta)=\Omega(\eta)([19]$, Chapter V), so that the dynamical system $(\Omega(\eta), T)$ is well-defined.

Given a bijective real function $f: \mathcal{A} \mapsto \mathbb{R}$, we associate a sequence in $l^{\infty}(\mathbb{Z})$ to each $\omega=\left(\omega_{n}\right)_{n \in \mathbb{Z}} \in \Omega$ by $\left(f\left(\omega_{n}\right)\right)_{n \in \mathbb{Z}}$, i.e., we just replace letters by numbers, which we again denote by $\omega$, and call it a substitution potential. Therefore, every point $\omega \in \Omega$ defines a bounded self-adjoint operator $H_{\omega}$ on $l^{2}(\mathbb{Z})$ by

$$
\left(H_{\omega} u\right)_{n}=u_{n+1}+u_{n-1}+\omega_{n} u_{n} .
$$

Our goal is the investigation of the spectral properties of the Schrödinger operator (11) for a special class of substitutions defined below.

Let $M$ be the matrix of the substitution $\xi$ whose entries $M_{a b}$ are given by the number of occurrences of the symbol $a$ in $\xi(b)$, for $a, b \in \mathcal{A}$. Recall that a matrix $M$ is primitive if all entries in a power of $M$ are strictly positive. A substitution rule $\xi$ is called primitive if its corresponding substitution matrix is primitive. It is known that for primitive substitution the subshift dynamical system $(\Omega, T)$ is strictly ergodic (see [19], Chapter V).

Here we consider a subclass of the following collection of substitutions $\zeta=\zeta_{N}$, for each $N \geq 1$, on $\mathcal{A}=\{a, b\}$, given by

$$
\zeta(a)=\underbrace{a \ldots a}_{A_{1}} \underbrace{b \ldots b}_{B_{1}} \underbrace{a \ldots a}_{A_{2}} \cdots \underbrace{b \ldots b}_{B_{N}} \underbrace{a \ldots a}_{A_{N+1}}, \quad \zeta(b)=b,
$$

with $A_{j}, B_{j} \geq 1, j=1, \cdots, N$, and $A_{N+1} \geq 1$ being the number of factors in each block. Such substitution is clearly nonprimitive, for any positive power of its matrix of substitution has the form

$$
\left[\begin{array}{ll}
A & 0 \\
B & 1
\end{array}\right]
$$

for some positive integers $A, B$. 
It is possible to generate periodic sequences with appropriate choices of $\zeta$ in (2); for instance, the substitution $\zeta(a)=a b b a, \zeta(b)=b$. The aperiodicity of the substitution sequences is significant for our spectral results; the following lemma presents a very simple and rather general condition on $\zeta$ assuring that $\eta$ is not periodic.

Lemma 1. If $A_{j} \geq 2$ for some $1 \leq j \leq(N+1)$, then the resulting substitution sequence $\eta$ is not ultimately periodic.

Proof. It is possible to restrict the argument for strictly positive index of $\eta$, i.e., to $\bar{\eta}$. For notational convenience we suppose that $A_{1}=2$; the other cases can be handled in a similar way. Notice first that all (constant) blocks of $a$ 's (and $b$ 's) in $\eta$ are finite. Suppose that $\eta$ is periodic with minimal period $\tau \geq 2$ ( $\eta$ is clearly nonconstant) and denote the first minimal period block, represented by the first $\tau$ letters of $\bar{\eta}$, by $B$. Let $n_{c}$ be the unique integer such that $(|Q|$ denotes the length of $Q \in \mathcal{A}^{*}$, e.g., $|B|=\tau$; it is also supposed that $\left.\zeta^{0}(a)=a\right)$

$$
\left|\zeta^{n_{c}-1}(a)\right|<\tau \leq\left|\zeta^{n_{c}}(a)\right| \text {. }
$$

The first entries of $\bar{\eta}$ are

$$
\zeta^{n_{c}+1}(a)=\zeta^{n_{c}}(a) \zeta^{n_{c}}(a) b \ldots b \zeta^{n_{c}}(a) \ldots \zeta^{n_{c}}(a) \cdots b \ldots b \zeta^{n_{c}}(a) \ldots \zeta^{n_{c}}(a) .
$$

The choice of $n_{c}$ entails that, besides at position 1 , a new period block $B$ starts at position $\left(\left|\zeta^{n_{c}}(a)\right|+1\right)$. Since the period block $B$ cannot appear shifted by any amount different from an integer multiple of $\tau$ (this would force a constant sequence $\bar{\eta}$ ), one sees that $\left|\zeta^{n_{c}}(a)\right|$ is an integer multiple of $\tau$. Therefore, a new period block $B$ should start at position $\left(2\left|\zeta^{n_{c}}(a)\right|+1\right)$, but this cannot occur since $B$ starts with the letter $a$ and at position $\left(2\left|\zeta^{n_{c}}(a)\right|+1\right)$ of $\bar{\eta}$ there is a $b$. Therefore $\bar{\eta}$ (and consequently $\eta$ ) is not periodic.

Suppose now that $\eta$ is ultimately periodic, so that there is an integer $k \geq 1$ such that $\eta$ is periodic, with period $\tau$, after its $k$ th position $(k \geq 2)$. Choose an integer $m$ such that $\left|\zeta^{m}(a)\right|>k$ and $\left|\zeta^{m}(a)\right|>\tau$. The first entries of $\bar{\eta}$ are

$$
\zeta^{m+1}(a)\left[\zeta^{m+1}(a)\right] b \ldots b \zeta^{m+1}(a) \ldots \zeta^{m+1}(a) \ldots b \ldots b \zeta^{m+1}(a) \ldots \zeta^{m+1}(a),
$$

and writing out the above second block $\zeta^{m+1}(a)$ one gets, for the beginning of $\bar{\eta}$,

$$
\zeta^{m+1}(a)\left[\zeta^{m}(a) \zeta^{m}(a) b \ldots b \zeta^{m}(a) \ldots \zeta^{m}(a) \ldots b \ldots b \zeta^{m}(a) \ldots \zeta^{m}(a)\right] b \ldots b .
$$

The choice of $m$ implies that a minimal period block $B$ starts at position $\left(\zeta^{m+1}(a)\right.$ +1 ) and also at $\left(\left|\zeta^{m+1}(a)\right|+\left|\zeta^{m}(a)\right|+1\right)$, and with the letter $a$. The same argument used above implies that another period block $B$ should start at position $\left(\left|\zeta^{m+1}(a)\right|+2\left|\zeta^{m}(a)\right|+1\right)$, i.e., $B$ should start with a $b$. This contradiction shows that $\bar{\eta}$ and $\eta$ are not ultimately periodic.

Although the substitution $\zeta$ is not primitive, it will be possible to adapt some techniques used for the primitive case in order to prove spectral results for (1), with potentials along the hull of a subclass $\zeta$ of (2) (to be specified later), always assuming from now on that:

(i) $\Omega_{\zeta}$ denotes the hull of the substitution $\zeta$ defined by (2), so that $\left(\Omega_{\zeta}, T\right)$ indicates the subshift dynamical system associated to $\zeta$.

(ii) The function $f: \mathcal{A} \mapsto \mathbb{R}$ defining the substitution potential is bijective (in order to avoid constant potentials). 
Some studies of the spectral properties of (1), with potentials $\left(\omega_{n}\right)$ generated by $\zeta_{N}$ satisfying $(i)$ and $(i i)$, will rely upon dynamical features of $\left(\Omega_{\zeta}, T\right)$ and an adaptation of the so-called Gordon argument (see, for instance, [9] and Section 10.2 of [18]); these are the contents of Propositions 1, 20 and 4 whose proofs are postponed to other sections of this work. However, they will be used below to present our spectral results, which are summarized in two theorems.

Proposition 1. The subshift dynamical system $\left(\Omega_{\zeta}, T\right)$ is strictly ergodic. We shall denote its unique probability ergodic measure by $\mu$.

From general arguments presented, for example, in Queffélec's book 19, Chapter $\mathrm{V}$, it follows from the strict ergodicity of $\left(\Omega_{\zeta}, T\right)$ that every word from $\mathcal{A}^{*}$ occurring in some $\omega \in \Omega_{\zeta}$ also occurs in every $\omega^{\prime} \in \Omega_{\zeta}$, and with a well-defined and strictly positive frequency.

As usual, we shall indicate the spectrum of a self-adjoint operator $H$ by $\sigma(H)$, and by $\sigma_{a c}(H)$ and $\sigma_{p}(H)$ the absolutely continuous and point spectrum of $H$, respectively. Recall that a generic set is a dense $G_{\delta}$ (countable intersections of open sets).

The above dynamical properties will imply the following spectral result:

Proposition 2. If $A_{j} \geq 2$ for some $1 \leq j \leq(N+1)$, then $\sigma_{a c}\left(H_{\omega}\right)=\emptyset$ for any $\omega \in \Omega_{\zeta}$.

A curious property of some sequences is palindromicity, which plays an important role in the exclusion of eigenvalues for discrete Schrödinger operators (10). A word in $\mathcal{A}^{*}$ is called a palindrome [20] 12] if it is the same when read backwards, and an element of $\mathcal{A}^{\mathbb{Z}}$ is palindromic if it contains arbitrary long palindromes. A compact and $T$-invariant minimal subset of $\mathcal{A}^{\mathbb{Z}}$ is called palindromic if it contains one palindromic sequence.

Proposition 3. If $\Omega_{\zeta}$ is palindromic, or if $\zeta(a)$ is a palindrome, then there exists a generic set $\Upsilon \subset \Omega_{\zeta}$ such that $\sigma_{p}\left(H_{\omega}\right)=\emptyset$ for any $\omega \in \Upsilon$.

Proof. Since the proof is essentially contained in 12 we just sketch it. If $\zeta(a)$ is a palindrome, then $\zeta(w)$ is also a palindrome for any palindrome $w$, so that a $\zeta$-fixed point $\eta$ has palindromes of arbitrary long length and $\Omega_{\zeta}$ is palindromic. Therefore the second alternative hypothesis in the proposition implies the first one which, by its turn, guarantees the presence of a dense set $\mathcal{S} \subset \Omega_{\zeta}$ of strong palindromes with $\sigma_{p}\left(H_{\omega}\right)=\emptyset$ for any $\omega \in \mathcal{S}$ (Proposition 2.1 and Theorem 5.1 of [12]). The generic property in the proposition follows by the set of results known as Wonderland Theorem [21] (see also Theorem 7.1 in [12]).

Our motivating case was the particular substitution $\zeta^{(p)}: \mathcal{A} \rightarrow \mathcal{A}^{*}, \zeta^{(p)}(a)=$ aabaa, $\zeta^{(p)}(b)=b$, discussed in [17. According to Lemma 1 1 and Proposition 1 this is an example of a nonperiodic substitution sequence of the type (2) for which $\Omega_{\zeta^{(p)}}$ is palindromic, since $\zeta^{(p)}(a)$ is a palindrome.

Theorem 1. Suppose $A_{j} \geq 2$ for some $1 \leq j \leq(N+1)$. If $\Omega_{\zeta}$ is palindromic, or if $\zeta(a)$ is a palindrome, then there exists a generic set $\Upsilon \subset \Omega_{\zeta}$ such that for any $\omega \in \Upsilon$ the operator $H_{\omega}$ has purely singular continuous spectrum.

Proof. Combine Propositions 2 and 3 . 
Besides palindromicity, another way to exclude point spectrum is via the following adaptation of Gordon's argument [9, 14, 7, [5, 6, 18].

Proposition 4. If either $\left(A_{1}+A_{N+1}\right) \geq 4$ or $A_{j} \geq 4$ for some $1 \leq j \leq(N+1)$ (or both), then there exists a measurable set $\Xi \subset \Omega_{\zeta}$, with $\mu(\Xi)=1$, such that $\sigma_{p}\left(H_{\omega}\right)=\emptyset$ for any $\omega \in \Xi$.

Theorem 2. If either $\left(A_{1}+A_{N+1}\right) \geq 4$ or $A_{j} \geq 4$ for some $1 \leq j \leq(N+1)$, then there exists a measurable set $\Xi \subset \Omega_{\zeta}$, with $\mu(\Xi)=1$, such that for any $\omega \in \Xi$ the operator $H_{\omega}$ has purely singular continuous spectrum.

Proof. Combine Propositions 2 and 4.

The above theorems present (very easy to verify) sufficient conditions for the occurrence of singular continuous spectra. For example, the substitutions $a \rightarrow$ abaaa, $a \rightarrow$ abbaaaaba, $a \rightarrow$ aaabababba ( $b \rightarrow b$ in all cases) result in pure singular continuous spectrum of $H_{\omega}$ for $\omega$ in sets of total invariant measure in the respective hulls. The palindromic nonprimitive substitution $\zeta(a)=a b a a b a$ in (2) gives rise to singular continuous spectrum for the operator (1) in a generic set of potentials in its hull; we do not have any results on the measure theoretical side in this case. The operator $H_{\omega}$, with potentials in the hull of the substitution $\zeta^{(p)}(a)=a a b a a$, has pure singular continuous spectrum for both in a generic set and in a set of full invariant measure of its hull (notice that this improves results previously obtained in 17).

The remainder of this work is organized as follows. In Section 2 we prove Propositions 1 and 2, and in Section 3 we give the proof of Proposition 4. In the final section we present additional remarks.

\section{StRict ERGODICITY}

In this section we prove Propositions 1 and 2. Before proceeding to the proof of Proposition 1, we recall that $|Q|$ is the length of $Q \in \mathcal{A}^{*}$ and say that $\operatorname{such} Q$ is called a $k$-block in $\omega \in \Omega_{\zeta}$ if $Q$ is a word contained in $\omega$ and $|Q|=k$. If $P$ and $Q$ belong to $\mathcal{A}^{*}, \#_{P} Q$ denotes the number of occurrences of $P$ in $Q$. An important point in Proposition 1 is to show that

$$
\lim _{j \rightarrow \infty} \frac{\#_{\zeta^{n}(a)}\left(\left|\zeta^{n+j}(a)\right| \text {-block }\right)}{\left|\zeta^{n+j}(a)\right|}
$$

exists, is nonzero and independent of the $\left|\zeta^{n+j}(a)\right|$-block considered; this implies that $\left(\Omega_{\zeta}, T\right)$ is uniquely ergodic $([23]$, Section 4.5$)$. To perform the required estimates, we define $f_{N+1}=\sum_{i=1}^{N+1} A_{i}$ and $h_{N}=\sum_{i=1}^{N} B_{i}$; the $j$ th power of $f_{N+1}$ will be denoted by $f_{N+1}^{j}$ and the ratio

$$
R_{j}=\left(f_{N+1}^{j}-1\right) /\left(f_{N+1}-1\right) ;
$$

notice that $f_{N+1} \geq 2$.

Lemma 2. The iterates of the substitution $\zeta$ satisfy:

(a) $\left|\zeta^{j}(a)\right|=f_{N+1}^{j}+h_{N} R_{j}$,

(b) $\quad \#_{a}\left(\zeta^{j}(a)\right)=f_{N+1}^{j}$. 
Proof. (a) It is clear that $|\zeta(a)|=f_{N+1}+h_{N}$ and

$$
\begin{aligned}
\left|\zeta^{2}(a)\right| & =f_{N+1}\left(f_{N+1}+h_{N}\right)+h_{N} \\
& =f_{N+1}^{2}+h_{N}\left(f_{N+1}+1\right) ;
\end{aligned}
$$

an induction argument gives

$$
\begin{aligned}
\left|\zeta^{j}(a)\right| & =f_{N+1}^{j}+h_{N}\left[f_{N+1}^{j-1}+\cdots+1\right] \\
& =f_{N+1}^{j}+h_{N} R_{j} .
\end{aligned}
$$

(b) This is a simple induction.

Define $C_{A}$ and $C_{B_{k}}$ by

$$
\begin{gathered}
\# \zeta_{\zeta^{n}(a)}\left(\zeta^{n}(a) \zeta^{n}(a)\right)=2+C_{A}, \\
\#_{\zeta^{n}(a)}(\zeta^{n}(a) \underbrace{b \ldots b}_{B_{k}} \zeta^{n}(a))=2+C_{B_{k}}, \quad 1 \leq k \leq N .
\end{gathered}
$$

Lemma 3. If $C_{A}>0$, then $C_{B_{q}}=0 \quad \forall 1 \leq q \leq N$. Conversely, if $C_{B_{q}}>0$ for some $q$, then $C_{A}=0$.

Proof. If $C_{A}>0$ there exists $2 \leq j \leq N$ such that

$$
\underbrace{a \ldots a}_{A_{j}-A_{N+1}} \underbrace{b \ldots b}_{B_{j}} \underbrace{a \ldots a}_{A_{j+1}} \cdots \underbrace{a \ldots a}_{A_{N+1}+A_{1}} \cdots \underbrace{b \ldots b}_{B_{j-1}} \underbrace{a \ldots a}_{A_{j}-A_{1}}=\underbrace{a \ldots a}_{A_{1}} \underbrace{b \ldots b}_{B_{1}} \underbrace{a \ldots a}_{A_{2}} \cdots \underbrace{b \ldots b}_{B_{N}} \underbrace{a \ldots a}_{A_{N+1}} ;
$$

then

$$
A_{k}=\left\{\begin{array}{ll}
A_{k+j-1}, & k+j-1<N+1 \\
A_{1}+A_{N+1}, & k+j-1=N+1, \\
A_{k+j-1-N}, & k+j-1>N+1,
\end{array} \quad 2 \leq k \leq N,\right.
$$

and

$$
B_{m}=\left\{\begin{array}{ll}
B_{j+m-1}, & k+j-1 \leq N, \\
B_{j+m-1-N}, & k+j-1>N,
\end{array} \quad 1 \leq m \leq N .\right.
$$

If $C_{B_{q}}>0$ for some $q$ there exists $2 \leq i \leq N$ such that

$$
\underbrace{a \ldots a}_{A_{i}} \underbrace{b \ldots b}_{B_{i}} \underbrace{a \ldots a}_{A_{i+1}} \cdots \underbrace{a \ldots a}_{A_{N+1}} \underbrace{b \ldots b}_{B_{q}} \underbrace{a \ldots a}_{A_{1}} \cdots \underbrace{b \ldots b}_{B_{i-2}} \underbrace{a \ldots a}_{A_{i-1}}=\underbrace{a \ldots a}_{A_{1}} \underbrace{b \ldots b}_{B_{1}} \underbrace{a \ldots a}_{A_{2}} \cdots \underbrace{b \ldots b}_{B_{N}} \underbrace{a \ldots a}_{A_{N+1}} .
$$

Then

$$
A_{l}=\left\{\begin{array}{ll}
A_{l+i-1}, & l+i-1 \leq N+1, \\
A_{l+i-1-(N+1)}, & l+i-1>N+1,
\end{array} \quad 1 \leq l \leq N\right.
$$

and

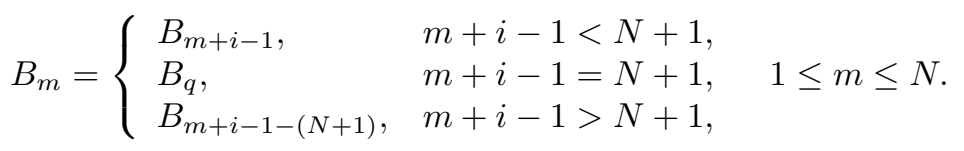

If conditions (3) and (5) are simultaneously satisfied we have (the number over the equal sign indicates the equation used in each step)

$$
\left(A_{1}+A_{N+1}\right) \stackrel{(3)}{=} A_{N-j+2} \stackrel{(5)}{=} A_{N-j+i+1} \stackrel{(3)}{=} A_{i} \stackrel{(5)}{=} A_{1}
$$

if $j \geq i$, and

$$
\left(A_{1}+A_{N+1}\right) \stackrel{(3)}{=} A_{N-j+2} \stackrel{(5)}{=} A_{i-j} \stackrel{(3)}{=} A_{i-1} \stackrel{(5)}{=} A_{N+1}
$$


if $j<i$. Since $A_{N+1}>0$ and $A_{1}>0$, we have obtained contradictions and the lemma is proven.

We remark that the maximum possible values of $C_{A}$ and $C_{B_{q}}$ are $(N-1)$ and $N$, respectively (and each of them occurs for suitable choices of $\zeta_{N}$ ).

Proof of Proposition 1. To begin with we show that $\left(\Omega_{\zeta}, T\right)$ is uniquely ergodic. Due to Lemma 3 it is enough to consider two cases:

(i) $\#_{\zeta^{n}(a)}\left(\zeta^{n}(a) \zeta^{n}(a)\right)=2+C_{A}, \quad \#_{\zeta^{n}(a)}\left(\zeta^{n}(a) B_{k} \zeta^{n}(a)\right)=2$;

(ii) $\#_{\zeta^{n}(a)}\left(\zeta^{n}(a) \zeta^{n}(a)\right)=2, \quad \#_{\zeta^{n}(a)}\left(\zeta^{n}(a) B_{k} \zeta^{n}(a)\right)=2+C_{B_{k}}$.

Notice that both cases include $C_{A}=C_{B_{k}}=0$, for any $k$, as particular instances.

In case (i), by Lemma 2 ,

$$
\# \zeta^{n}(a)\left(\zeta^{n+j}(a)\right)=f_{N+1}^{j}+\left(f_{N+1}-(N+1)\right) C_{A} R_{j},
$$

and by counting the occurrences of $\zeta^{n}(a)$ in $\left|\zeta^{n}(a)\right|$-blocks we find

$$
\begin{array}{r}
f_{N+1}^{j}+\left(f_{N+1}-(N+1)\right) C_{A} R_{j}-C_{A}-1 \leq \#_{\zeta^{n}(a)}\left(\left|\zeta^{n+j}(a)\right| \text {-block }\right) \\
\leq f_{N+1}^{j}+\left(f_{N+1}-(N+1)\right) C_{A} R_{j}+C_{A}-1,
\end{array}
$$

so that

$$
\begin{aligned}
\lim _{j \rightarrow \infty} \frac{\#_{\zeta^{n}(a)}\left(\left|\zeta^{n+j}(a)\right| \text {-block }\right)}{\left|\zeta^{n+j}(a)\right|} & =\lim _{j \rightarrow \infty} \frac{f_{N+1}^{j}+\left(f_{N+1}-(N+1)\right) C_{A} R_{j}}{f_{N+1}^{j+n}+h_{N} R_{j+n}} \\
& =\frac{f_{N+1}+\left(f_{N+1}-(N+1)\right) C_{A}-1}{f_{N+1}^{n}\left[f_{N+1}+h_{N}-1\right]}>0 .
\end{aligned}
$$

In case (ii) there is an integer $M_{k} \geq 0$, which depends on $N$, such that $\left(M_{k}\right.$ takes into account the existence of eventual $B_{l}=B_{k}$ for $k \neq l$ )

$$
\#_{\zeta^{n}(a)}\left(\zeta^{n+j}(a)\right)=f_{N+1}^{j}+M_{k} R_{j} .
$$

Again by counting the occurrences we find

$$
\begin{aligned}
f_{N+1}^{j}+M_{k} R_{j}-C_{B_{k}}-1 & \leq \#_{\zeta^{n}(a)}\left(\left|\zeta^{n+j}(a)\right| \text {-block }\right) \\
& \leq f_{N+1}^{j}+M_{k} R_{j}+C_{B_{k}}-1,
\end{aligned}
$$

so that

$$
\begin{aligned}
\lim _{j \rightarrow \infty} \frac{\#_{\zeta^{n}(a)}\left(\left|\zeta^{n+j}(a)\right| \text {-block }\right)}{\left|\zeta^{n+j}(a)\right|} & =\lim _{j \rightarrow \infty} \frac{f_{N+1}^{j}+M_{k} R_{j}}{f_{N+1}^{j+n}+h_{N} R_{j+n}} \\
& =\frac{f_{N+1}+M_{k}-1}{f_{N+1}^{n}\left[f_{N+1}+h_{N}-1\right]}>0 .
\end{aligned}
$$

(If there are other $C_{B_{q}} \neq 0, B_{q} \neq B_{l}$ for $k \neq l$, just replace $M_{k}$ by $\sum_{q} M_{q}$ in the above average, with the sum over $q$ such that $B_{q} \neq B_{l}$.) Therefore $\left(\Omega_{\zeta}, T\right)$ is uniquely ergodic.

Now we show that $\left(\Omega_{\zeta}, T\right)$ is minimal. Given a word $B \in \mathcal{A}^{*}$ occurring in $\eta$, there is an $m \in \mathbb{N}$ such that $B \subset \zeta^{m}(a)$. Now, $\zeta^{m}(a)$ occurs in $\eta$ with bounded gaps (with bound $\leq \max \left\{B_{1}, B_{2}, \cdots, B_{N}\right\}+\left|\zeta^{m}(a)\right|$ ); this implies that $\eta$ is almost periodic, and since from the very definition of $\zeta$ it follows that $\eta$ is also recurrent, one concludes that $\left(\Omega_{\zeta}, T\right)$ is minimal ([19], Section IV.2). Therefore $\left(\Omega_{\zeta}, T\right)$ is strictly ergodic. 
Proof of Proposition 2. The conclusions of Proposition 2 will follow from Proposition 1 and two additional results. The first one is due to Last and Simon [22] which, in this setting, states that for strictly ergodic potentials the absolutely continuous spectrum is independent of $\omega \in \Omega_{\zeta}$. The second result we use is due to Kotani [13]: for nonperiodic ergodic potentials taking only a finite number of values the set of potentials with no absolutely continuous spectrum has full ergodic measure. By Proposition 1 our (nonperiodic) substitution potentials are strictly ergodic, so by Last-Simon $\sigma_{a c}\left(H_{\omega}\right)$ is constant on $\Omega_{\zeta}$; since by Kotani and Lemma 1 there are potentials with $\sigma_{a c}\left(H_{\omega}\right)=\emptyset$, it follows that $\sigma_{a c}\left(H_{\omega}\right)=\emptyset$ for any $\omega \in \Omega_{\zeta}$.

\section{A Gordon type argument}

Now we will show that if $\left(A_{1}+A_{N+1}\right) \geq 4$ or $A_{j} \geq 4$ for some $1 \leq j \leq N+1$, then $\sigma_{p}\left(H_{\omega}\right)=\emptyset$ for $\omega$ in a set of full invariant measure $\mu$ in the hull $\Omega_{\zeta}$, i.e., we prove Proposition 4. We shall follow a Gordon type argument similar to the one used in $9,14,7,5,6,18$.

Let's first sketch the idea of the proof. For a fixed $\omega \in \mathcal{A}^{\mathbb{Z}}$, if there is a sequence $m \rightarrow \infty$ such that, for each such $m$,

$$
\omega_{k-m}=\omega_{k}=\omega_{k+m}, \quad 1 \leq k \leq m,
$$

then the Schrödinger operator (1) has no eigenvalues; this is the Gordon argument (see the above references). It will be shown that, under the assumptions of Proposition 4 , the measurable sets

$$
B(n) \equiv\left\{\omega \in \Omega_{\zeta}: \omega_{\left(k-\left|\zeta^{n}(a)\right|\right)}=\omega_{k}=\omega_{\left(k+\left|\zeta^{n}(a)\right|\right)}, 1 \leq k \leq\left|\zeta^{n}(a)\right|\right\}
$$

satisfy

$$
\mu\left(\limsup _{n \rightarrow \infty} B(n)\right)>0,
$$

and since $\lim \sup _{n \rightarrow \infty} B(n)$ is contained in the shift invariant set $\left\{\omega \in \Omega_{\zeta}: \sigma_{p}\left(H_{\omega}\right)\right.$ $=\emptyset\}$, it will follow, by an argument in ergodic theory, that the latter set has full invariant measure.

If $\eta$ is the bilateral substitution sequence generated by $\zeta$, it is then natural to investigate the sets

$$
J(n) \equiv\left\{\text { distinct words of } \eta \text { of the form } v v v \text { with }|v|=\left|\zeta^{n}(a)\right|\right\}
$$

and, in particular, the values of their measures $\mu(J(n))$, which can be recovered by ergodic averages. The following lemmas will be used to estimate such averages.

Lemma 4. If $\left(A_{1}+A_{N+1}\right) \geq 4$ or $A_{j} \geq 4$ for some $1 \leq j \leq N+1$, then for all $n \geq 1 \# J(n)$ (the cardinality of $J(n)$ ) satisfies

$$
B_{1}+A_{2}\left|\zeta^{n-1}(a)\right| \leq \# J(n) \leq\left|\zeta^{n}(a)\right| .
$$

Proof. The occurrence of aaaa in $\eta$ yields existence of blocks

$$
\zeta^{n}(a) \zeta^{n}(a) \zeta^{n}(a) \zeta^{n}(a)
$$

that contain all different blocks of the form vvv, with $|v|=\left|\zeta^{n}(a)\right|$. Thus we consider cyclic permutations of $\zeta^{n}(a)$ to determine the number of different blocks $v v v$. For each block $u_{1} u_{2} \cdots u_{l}$ let

$$
P\left(u_{1} u_{2} \cdots u_{l}\right)=u_{2} u_{3} \cdots u_{l} u_{1} .
$$


Thus

$$
\# J(n)=\min \left\{i \geq 1: \zeta^{n}(a)=P^{i}\left(\zeta^{n}(a)\right)\right\} \leq\left|\zeta^{n}(a)\right| .
$$

For a general substitution of type (2), by successively applying $P$ to $\zeta(a)$, the first opportunity to recover $\zeta(a)$ is (see the examples at the end of this proof)

$$
\underbrace{a \ldots a}_{A_{2}-A_{N+1}} \underbrace{b \ldots b}_{B_{2}} \cdots \underbrace{a \ldots a}_{A_{1}+A_{N+1}} \underbrace{b \ldots b}_{B_{1}} \underbrace{a \ldots a}_{A_{2}-A_{1}} .
$$

Hence we have $B_{1}+A_{2} \leq \# J(1) \leq|\zeta(a)|$. By the very definition of the substitution, this property generalizes for any positive integer $n$

$$
B_{1}+A_{2}\left|\zeta^{n-1}(a)\right| \leq \# J(n) \leq\left|\zeta^{n}(a)\right| .
$$

Both extreme conditions can occur. For example, if all $B_{j}$ are pairwise different or $A_{j} \neq A_{1}+A_{N+1}, \forall 2 \leq j \leq N$, one gets $\# J(n)=\left|\zeta^{n}(a)\right|$; while the case in which all $B_{j}$ are equal and $A_{j}=A_{1}+A_{N+1}, \forall 2 \leq j \leq N$, one gets \#J(n)= $B_{1}+A_{2}\left|\zeta^{n-1}(a)\right|$.

Lemma 5. If vvv occurs in $\eta$ with $|v|=\left|\zeta^{n}(a)\right|$, then

$$
\begin{aligned}
\#_{v v v} \zeta^{n+j}(a) & \geq \#_{a a a a} \zeta^{j}(a) \\
& =f_{N+1}^{j-1} \sum_{\left\{i: A_{i} \geq 4\right\}}\left(A_{i}-3\right)+R_{j-1} E_{N} \sum_{i=1}^{N+1}\left(A_{i}-1\right),
\end{aligned}
$$

where

$$
E_{N}=\left\{\begin{array}{cl}
0 & \text { if } A_{1}+A_{N+1}<4, \\
1 & \text { if } A_{1}=A_{N+1}=2, \\
3 & \text { if } \min \left\{A_{1}, A_{N+1}\right\} \geq 3, \\
\min \left\{A_{1}, A_{N+1}\right\} & \text { otherwise. }
\end{array}\right.
$$

Proof. By the construction of the blocks $v v v$ with $|v|=\left|\zeta^{n}(a)\right|$, in the proof of Lemma 4, we have

$$
\#_{v v v} \zeta^{n+j}(a) \geq \#_{\zeta^{n}(a a a a)} \zeta^{n+j}(a)=\#_{a a a a} \zeta^{j}(a) .
$$

Direct computation gives \# $\#_{a a a} \zeta(a)=\sum_{\left\{i: A_{i} \geq 4\right\}}\left(A_{i}-3\right)$, and

$$
\#_{\text {aaaa }} \zeta^{2}(a)=f_{N+1} \sum_{\left\{i: A_{i} \geq 4\right\}}\left(A_{i}-3\right)+E_{N} \sum_{i=1}^{N+1}\left(A_{i}-1\right) .
$$

Then, by induction one concludes that

$$
\begin{aligned}
\#_{a a a a} \zeta^{j}(a) & =f_{N+1}^{j-1} \sum_{\left\{i: A_{i} \geq 4\right\}}\left(A_{i}-3\right)+\left[f_{N+1}^{j-2}+\cdots+1\right] E_{N} \sum_{i=1}^{N+1}\left(A_{i}-1\right) \\
& =f_{N+1}^{j-1} \sum_{\left\{i: A_{i} \geq 4\right\}}\left(A_{i}-3\right)+R_{j-1} E_{N} \sum_{i=1}^{N+1}\left(A_{i}-1\right) .
\end{aligned}
$$

The Borel $\sigma$-algebra in $\left(\Omega_{\zeta}, T\right)$ is generated by the cylinders

$$
\left[u_{0} \cdots u_{l-1}\right]_{[m, \cdots, m+l-1]} \equiv\left\{\omega \in \Omega_{\zeta}: \omega_{m+i}=u_{i}, 0 \leq i \leq l-1\right\} .
$$


Since $\Omega_{\zeta}$ is strictly ergodic, it is known that (19], Section V.4) the unique invariant probability measure $\mu$ satisfies

$$
d\left(u_{0} \cdots u_{l-1}\right) \equiv \mu\left(\left[u_{0} \cdots u_{l-1}\right]_{[m, \cdots, m+l-1]}\right)=\lim _{n \rightarrow \infty} \frac{\# u_{0} \cdots u_{l-1} \zeta^{n}(a)}{\left|\zeta^{n}(a)\right|} .
$$

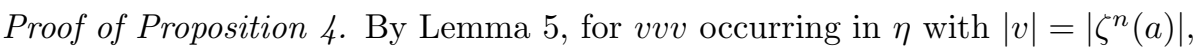

$$
\begin{aligned}
d(v v v) & =\lim _{j \rightarrow \infty} \frac{\#_{v v v} \zeta^{n+j}(a)}{\left|\zeta^{n+j}(a)\right|} \\
& \geq \lim _{j \rightarrow \infty} \frac{\#_{a a a a} \zeta^{j}(a)}{\left|\zeta^{n+j}(a)\right|} \\
& =\lim _{j \rightarrow \infty} \frac{f_{N+1}^{j-1} \sum_{\left\{i: A_{i} \geq 4\right\}}\left(A_{i}-3\right)+R_{j-1} E_{N} \sum_{i=1}^{N+1}\left(A_{i}-1\right)}{f_{N+1}^{n+j}+R_{n+j} h_{N}} \\
& =\frac{d_{N}}{f_{N+1}^{n+1}\left(f_{N+1}+h_{N}-1\right)},
\end{aligned}
$$

with $d_{N}=\left[f_{N+1}-1\right] \sum_{\left\{i: A_{i} \geq 4\right\}}\left(A_{i}-3\right)+E_{N} \sum_{i=1}^{N+1}\left(A_{i}-1\right)$. Since the set $B(n)$ is the finite union of disjoint cylinder sets $[v v v]_{I_{n}}$, with $v v v \in J(n)$, where $I_{n}=$ $\left[1-\left|\zeta^{n}(a)\right|, 2\left|\zeta^{n}(a)\right|\right]$,

$$
\begin{aligned}
\mu(B(n)) & =\sum_{v v v \in J(n)} \mu\left([v v v]_{I_{n}}\right) \\
& =\sum_{v v v \in J(n)} d(v v v) \\
& \geq\left[B_{1}+A_{2}\left(f_{N+1}^{n-1}+h_{N} R_{n-1}\right)\right] d(v v v) .
\end{aligned}
$$

By writing out $d(v v v)$ one gets

$$
\lim _{n \rightarrow \infty} \mu(B(n)) \geq \frac{A_{2}\left(f_{N+1}+h_{N}-1\right) d_{N}}{f_{N+1}^{4}-2 f_{N+1}^{3}+f_{N+1}^{2}+\left(f_{N+1}^{2} h_{N}\right)\left(f_{N+1}-1\right)}>0 .
$$

Noticing that [7, 9]

$$
\limsup B(n) \subseteq C_{p} \equiv\left\{\omega \in \Omega_{\zeta}: \sigma_{p}\left(H_{\omega}\right)=\emptyset\right\},
$$

$C_{p}$ is subshift invariant, $\mu$ is ergodic, and

$$
\mu\left(C_{p}\right) \geq \mu(\lim \sup B(n)) \geq \lim \mu(B(n))>0,
$$

one concludes that $\sigma_{p}\left(H_{\omega}\right)=\emptyset \mu$-a.e. $\omega \in \Omega_{\zeta}$, since subshift invariants sets in $\left(\Omega_{\zeta}, T\right)$ have either null or full ergodic measure.

\section{Final Remarks}

1. Notice that the integer 1 is always an eigenvalue of the matrix of substitution $M(\zeta)$, and the other eigenvalue is the positive integer $\sum_{k=1}^{N+1} A_{k}>1$, clearly a non-Pisot number. Notice also that besides nonprimitive, $\zeta$ is not irreducible.

2. We remark that Propositions 3 and 4, used to exclude point spectrum, can be adapted for ultimately periodic sequences. The nonperiodicity were important here for the exclusion of the absolutely continuous spectrum in Proposition 2,

3. The generalization of the above results for the case $\zeta(b)=b b \ldots b$ in (2), with more than one factor, is not direct, since in this case there are words composed only of $b$ 's of arbitrary long length and $\left(\Omega_{\zeta}, T\right)$ is not strictly ergodic. 
4. As a direct corollary of our results it follows that for palindromic substitutions $\zeta$, with $A_{1}+A_{N+1} \geq 4$ or $A_{j} \geq 4$ for some $1 \leq j \leq(N+1)$, the spectrum $\sigma\left(H_{\omega}\right)$ is pure singular continuous for $\omega$ in a generic set as well as in a set of full invariant measure.

5. Another possibility to exclude point spectrum directly for the fixed-point sequence $\eta$ of the substitution $\zeta$ is to apply the techniques presented in references [14, [5].

6. Given a particular substitution $\zeta_{N}$ (under the hypotheses of Proposition 4 ) it is possible to find the value of $\lim _{n \rightarrow \infty} \mu(B(n))$. E.g., if all $B_{j}$ are pairwise different or $A_{j} \neq A_{1}+A_{N+1}, \forall j$, then this limit is given by

$$
\frac{\left(f_{N+1}+h_{N}-1\right) d_{N}}{f_{N+1}^{3}-2 f_{N+1}^{2}+f_{N+1}+\left(f_{N+1} h_{N}\right)\left(f_{N+1}-1\right)} \text {. }
$$

7. An open problem is to characterize the spectral type [19] of the autocorrelation measure for the nonperiodic substitution sequences generated by $\zeta_{N}$. How does it depend on $A_{1}, \cdots, A_{N+1}, B_{1}, \cdots, B_{N}$ ?

\section{REFERENCES}

[1] Axel, F., Gratias, D. (eds.), Beyond Quasicrystals, Berlin: Les Editions de Physique/Springer-Verlag: 1995. MR 97e:00004

[2] Bellissard, J.: Spectral properties of Schrödinger's operator with Thue-Morse potential. In: Luck, J.-M., Moussa, P., Waldschmidt, M. (eds.) Number Theory and Physics, Springer Proceedings in Physics, Vol. 47, Berlin: Springer 1990, pp 140-150. MR 92b:82007

[3] Bellissard, J., Bovier, A., Ghez, J.-M.: Spectral properties of a tight binding Hamiltonian with period doubling potential. Commun. Math. Phys. 135, 379-399 (1991). MR 91m:81042

[4] Bellissard, J., Bovier, A., Ghez, J.-M.: Gap labelling theorems for one dimensional discrete Schrödinger operators. Rev. Math. Phys. 4, 1-37 (1992). MR 93f:47090

[5] Bovier, A., Ghez, J.-M.: Spectral properties of one-dimensional Schrödinger operators with potentials generated by substitutions. Commun. Math. Phys. 158, 45-66 (1993). MR 94k:82064

[6] Bovier, A., Ghez, J.-M.: Erratum to Ref. [5, Commun. Math. Phys. 166, 431-432 (1994). MR 95j:82033

[7] Damanik, D.: Singular continuous spectrum for the period doubling Hamiltonian on a set of full measure. Commun. Math. Phys. 196, 477-483 (1998). MR 99i:81035

[8] Damanik, D.: Singular continuous spectrum for a class of substitution Hamiltonians. Lett. Math. Phys. 46, 303-311 (1998). MR 99m:81053

[9] Delyon, F., Petritis, D.: Absence of localization in a class of Schrödinger operators with quasiperiodic potential. Commun. Math. Phys. 103, 441-444 (1986). MR 87e:81030

[10] Delyon, F., Peyrière, J.: Recurrence of the eigenstates of a Schrödinger operator with automatic potential. J. Stat. Phys. 64, 363-368 (1991). MR 92h:82066

[11] Hof, A.: Some remarks on discrete aperiodic Schrödinger operators. J. Stat. Phys. 72, 13531374 (1993). MR 94j:82007

[12] Hof, A., Knill, O., Simon, B.: Singular continuous spectrum for palindromic Schrödinger operators. Commun. Math. Phys. 174, 149-159 (1995). MR 97c:47038

[13] Kotani, S.: Jacobi matrices with random potential taking finitely many values. Rev. Math. Phys. 1, 129-133 (1989). MR 91b:81023

[14] Sütő, A.: The spectrum of a quasiperiodic Schrödinger operator. Commun. Math. Phys. 111, 409-415 (1987). MR 88m:81032

[15] Sütö, A.: Singular Continuous Spectrum on a Cantor Set of Zero Lebesgue Measure for the Fibonacci Hamiltonian. J. Stat. Phys. 56, 525-531 (1989). MR 90e:82046

[16] Iochum, B., Testard, D.: Power law growth for the resistance in the Fibonacci model. J. Stat. Phys. 65, 715-723 (1991). MR 93f:82006

[17] de Oliveira, C. R., Lima, M. V.: A nonprimitive substitution Schrödinger operator with generic singular continuous spectrum. Rep. Math. Phys. 45, 431-436 (2000). CMP 2000:16 
[18] Cycon, H. L., Forese, R. G., Kirsch, W., Simon, B.: Schrödinger Operators. Berlin: SpringerVerlag, 1987. MR 88g:35003

[19] Queffélec, M.: Substitution Dynamical Systems - Spectral Analysis, LNM 1294. Berlin: Springer-Verlag, 1987. MR 89g:54094

[20] Wen, Z.-Y.: Singular words, invertible substitutions and local isomorphisms. In Ref. [1] pp. 433-440. MR 98g:11027

[21] Simon, B.: Operators with singular continuous spectrum: I. General operators. Ann. Math. 141, 131-145 (1995). MR 96a:47038

[22] Last, Y., Simon, B.: Eigenfunctions, transfer matrices, and absolutely continuous spectrum of one-dimensional Schrödinger operators. Invent. Math. 135, 329-367 (1999). MR 2000f:47060

[23] Petersen, K.: Ergodic Theory, Cambridge: University Press, 1983. MR 87i:28002

Departamento de Matemática - UfSCar, São Carlos, SP, 13560-970 Brazil

E-mail address: oliveira@dm.ufscar.br

Departamento de Matemática - UfSCar, São Carlos, SP, 13560-970 Brazil, and AFA, Pirassununga, SP, 13630-000 Brazil

E-mail address: lima@dm.ufscar.br 